\title{
Bioanalysis
}

\section{2th GCC Closed Forum: critical reagents; oligonucleotides; CoA; method transfer; HRMS; flow cytometry; regulatory findings; stability and immunogenicity}

The 12th GCC Closed Forum was held in Philadelphia, PA, USA, on 9 April 2018. Representatives from international bioanalytical Contract Research Organizations were in attendance in order to discuss scientific and regulatory issues specific to bioanalysis. The issues discussed at the meeting included: critical reagents; oligonucleotides; certificates of analysis; method transfer; high resolution mass spectrometry; flow cytometry; recent regulatory findings and case studies involving stability and nonclinical immunogenicity. Conclusions and consensus from discussions of these topics are included in this article.

First draft submitted: 9 May 2019; Accepted for publication: 29 May 2019; Published online: 19 July 2019

Keywords: certificate of analysis $\bullet$ critical reagents $\bullet$ CRO $\bullet$ flow cytometry $\bullet$ GCC $\bullet$ HRMS $\bullet$ method transfer • oligonucleotides $\bullet$ regulatory findings $\bullet$ stability and immunogenicity

The 12th Closed Forum of the Global Contract Research Organization (CRO) Council in Bioanalysis (GCC) was held on 9 April 2018 in Philadelphia, PA, USA. Founded in 2010 [1], the GCC unites bioanalytical representatives from international CROs to discuss scientific and regulatory issues in bioanalysis. Member companies submit topics affecting their unique realities in order to stimulate discussions and obtain consensus; thereafter, presented as conference reports and White Papers [2-15].

Nine topics were discussed at the 12th Closed Forum; each included results of a survey designed by topic leaders which was circulated to GCC member representatives and compiled for presentation. The topics and leaders were:

- Recent findings/483s - Nicki Hughes, Allan Xu, Bruce Stouffer, Rafiq Islam, Maria Caturla;

- Critical reagent characterization and handling for PK, biomarker and immunogenicity assays - Paul Rhyne, Chris Beaver;

- Oligonucleotide outsourcing: best approach for a CRO - Bruce Stouffer, Jessica St Charles, Eric Tewalt;

- 'What would you do?' A session of case studies - Chad Briscoe, Edward Tabler, Franklin Spriggs, Kelly Colletti;

- Challenges of method transfer for LCMS, hybrid ligand binding assay (LBA)/LCMS and LBA - Chad Briscoe, Nicki Hughes;

- Certificates of Analysis (COA) - Roger Hayes;

- High resolution mass spectrometry (HRMS) in regulated bioanalysis - Stephanie Cape, Pat Bennett;

- Integration and standardization of flow cytometry in bioanalysis - Pat Bennett, Vellalore Kakkanaiah;

- Classification of commercial kits to support regulated bioanalysis - Rafiq Islam.

This report summarizes survey results and discussions from the meeting for all topics except for the classification of commercial kits, which is discussed in a separate White Paper [16].

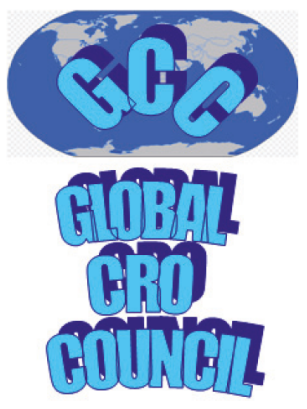

Chad Briscoe ${ }^{1}$, Nicola Hughes², Roger Hayes ${ }^{3}$ et al.

*Author for correspondence: wei@global-cro-council.org

Disclosure: Due to the equality principles of Global CRO Council for Bioanalysis (GCC), the authors are presented in alphabetical order of member company names, with the exception of the first 14 authors, who provided major contributions to the topics discussed as the topic leaders/speakers of the meeting.

The full list of author names and affiliations can be found at the end of the article 


\section{Discussion topics}

\section{Recent findings/483s}

Speakers presented recent experiences and findings from inspections performed by Brazilian

Health Regulatory Agency (ANVISA), European Medicines Agency (EMA) and US FDA.

One ANVISA inspection experience was presented. The CRO in question had specific standard operating procedures (SOPs) in place for bioanalysis of studies destined for ANVISA submission. However, the sponsor did not inform the CRO of the ANVISA filing until after the study began. As a result, findings were issued in order to meet the specific ANVISA Resolution requirements [17]. The first finding was regarding stability evaluations. The sponsor had used an alternate approach to calculate the stability results (e.g., two-thirds of samples with adequate deviation) but this was not accepted by ANVISA, as the requirement is that the stability must be assessed against nominal. The second finding referred to sponsor-requested formatting of the data, requiring reformatting to meet ANVISA requirements. Finally, ANVISA Resolution RDC 27 [17] does not allow re-analysis for which there is no clear reason other than that based on compartmental pharmacokinetics (PK) analysis. The CRO's general SOPs (not those designated for ANVISA submission) allowed PK repeats with a documented request from the sponsor. The CRO was not informed of the ANVISA filing, and limited PK repeats were performed at the sponsor's request and in accordance with the CRO's general SOPs. Based on these findings, it was concluded that sponsor requests should be carefully considered and discussed with the sponsor to evaluate regulatory compliance risks and clarity on the intended use of the data.

Two EMA inspections were discussed. The first discussion was around a joint inspection with FDA inspectors of a biosimilar program. The first major finding stated that it was not possible to verify the integrity of samples received by the laboratory as temperature monitoring devices were not included in sample shipments from clinical sites. The CRO was able to provide a shipping validation as well as documents confirming re-icing of samples by the shipping company. This was sufficient to confirm the integrity of samples for the study in question. However, EMA inspectors communicated their expectation that temperature-monitoring devices be used. The inspector was questioned about the bioanalytical laboratory receiving the finding since the clinical facility was responsible for shipping the samples, and the inspector stated that the laboratory was ultimately responsible for sample stability. Another CRO also discussed a similar experience with a Health Canada inspection where - although a temperature monitoring device was present in the shipment - the inspector expected a shipping validation as well, as they did not approve of shipping companies opening boxes containing study samples to replenish dry ice. The second major finding related to in-study cut-point criteria. The bioanalytical plan required the use of 50 predose samples to evaluate the in-study cut-point, which matched a recommendation from the 2017 White Paper in Bioanalysis [18]. However, the evaluation criteria were agreed upon a priori between the CRO and the sponsor only via email, and were not outlined directly in the bioanalytical plan, as required by the agency.

The second EMA case involved deficiency letters from the Danish, Spanish and Italian health authorities (HA) related to studies where samples were repeated as part of laboratory investigations: in other words, two instances of suspected sample name inversions and one instance of an incurred sample reanalysis sample differing by $>50 \%$ from the original value. The EMA bioanayltical method validation (BMV) guidance [19] allows for reanalysis to be considered as part of a laboratory investigation to identify possible reasons for abnormal results. In all instances cited above, the Health Authority stated that re-analysis was not permitted and the PK and statistical analysis would need to be performed with the original analysis. It should be noted that when this was done, the outcome of the studies did not change. Furthermore, in one case above, the Danish Health Authority cited the CRO because during the investigation, the validated number of freeze-thaw cycles was exceeded, despite the data not being reported. This example illustrates the difficulty CROs still have regarding attaining a balance between the need to perform laboratory investigations to ensure accurate and precise data and the prohibition on performing perceived PK repeats on anomalous study samples.

Three CROs presented information regarding FDA inspections. One CRO noted that over an inspection history of more than 20 years and over ten inspections, significant differences have been seen since 2009. The inspection frequency changed from an average of 2 years to 18 months. The duration of inspections increased as well, from 2-3 to 4-5 days, likely due to the fact that prior to 2009, two to four inspectors participated in the inspection; whereas, after 2009, only one inspector typically performed the inspection.

Observations from FDA inspections were related to calibration curves analyzing endogenous compounds, biosimilar immunogenicity assays and reporting of all validation batches as part of precision and accuracy. 
In the case of calibration curves analyzing endogenous compounds, the agency referred to the draft BMV guidance [20], which states that "the concentrations for the quality controls (QCs) should account for the endogenous concentrations in the biological matrix (i.e., additive)...". The CRO had not taken the endogenous level into account because the blank serum response was an extrapolated value and less than $20 \%$ of the lower limit of quantification of the method, and so thought to be negligible. Therefore, SOPs must have a procedure to ensure that endogenous concentrations of blank matrix are taken into account when calculating the calibration curve concentrations.

A biosimilar immunogenicity audit was performed by FDA, which extensively reviewed the approaches to biosimilar immunogenicity method validation and the use of one cut-point for both innovator and biosimilar molecules. It was strongly recommended by the agency to perform in-study cut-point evaluations and measure study sample results against the in-study cut-point. In addition, the reviewers focused on the overall variability of the assay, which included the variability (\%coeffecient of variation) of lysophosphatidylcholine inhibition and the variability of the assay signal when it was run multiple times. In summary, the FDA stated that the long term stability of the positive controls must be performed to cover the sample storage period, and more extensive validation should be performed in a Tier II assay.

Finally, deficiency letters and 483 s have been issued for not including all batches (passing and failing) in the precision and accuracy evaluation, as required by the FDA draft BMV [20].

\section{Critical reagent characterization and handling for PK, biomarker \& immunogenicity assays}

A survey consisting of ten questions was sent to GCC membership in order to determine how members define and manage critical reagents. The first step involved clarifying what could be considered a critical reagent. Overwhelmingly, antibody conjugates and target capture molecules were considered critical reagents for PK assays by $93 \%$ and $86 \%$ of respondents, respectively. Approximately $38 \%$ considered matrix and $14 \%$ considered assay buffers as critical reagents as well. For immunogenicity assays, respondents agreed that drug conjugates (89\%) and positive control antibodies (96\%) were critical reagents. Surprising to many, matrix pools were only considered critical reagents by $59 \%$ of respondents. Some other examples provided included precoated plates, MSD plates/reagents, stabilizers, derivatizing agents and additives. It was suggested that a tiered approach to managing critical reagents could be used, where reagents for Tier I assays like antibody conjugates, target capture molecules or positive control antibodies be strictly managed, and lower tier assays undergo a risk assessment to determine the criticality of the reagents.

When determining the optimal reagent, bioanalysts consider the performance of the assay (93\%), label to antibody ratio $(31 \%)$, affinity $(31 \%)$ and purity $(24 \%)$ as key aspects. For Tier I critical reagents, $93 \%$ of respondents believe there is a need to characterize critical reagents in their development and a further $90 \%$ believe they should be qualified in the assay prior to use. When respondents were asked if there should be different levels of characterization of reagents it was mentioned that it is often difficult to obtain sufficient characterization information or permission for upfront testing from sponsors on some reagents. For both PK and immunogenicity assays, $65 \%$ believed there should be different levels of characterization. When performing an evaluation on the level of characterization needed, bioanalysts should consider the tier of the assay and if different lots would be needed over the assay lifetime. Decisions should be documented. The tools used for characterization include using the LBA itself (79\%), HPLC (41\%), LCMS (21\%) and surface plasmon resonance (SPR; 10\%).

If additional lots of reagent are needed, $86 \%$ of respondents confirmed that the same characterization and qualification requirements are needed for subsequent lots. Only 68\% accept new lots that differ from the previous lots based on critical reagent analysis. As a criterion, 93\% stated that new lots must result in comparable performance in the assay as the previous lot, and $25 \%$ require only that reagent characteristics be comparable to the old lot.

\section{Oligonucleotide bioanalysis outsourcing: best approach for a CRO}

In recent years, there has been an increased focus on the use of oligonucleotides to treat diseases. Multiple types of oligonucleotides are being investigated in an array of different matrices using a variety of assay types. It has become necessary to establish assay parameters and limitations in order to define acceptance criteria for different assay formats. However, there remains uncertainty as to what aspects of regulatory guidance these assays should follow. In an effort to determine how the industry is adapting to this issue, a survey was sent to GCC membership, to which 34 members responded. Of these, $62 \%$ stated that their company currently conducts oligonucleotide bioanalysis, and of those who do not, $63 \%$ said they would in the future. Over the last 5 years, the number of 
Table 1. Platforms and criteria used to assay oligonucleotides.

\begin{tabular}{|lll|}
\hline Platform & Criteria & $\begin{array}{l}\text { Percent of respondents, more than one option possible } \\
(\%)\end{array}$ \\
\hline HPLC & $\leq 15(\leq 20 \%$ LOQ $)$ & 100 \\
& $\leq 20(\leq 25 \%$ LOQ $)$ & 14 \\
& $\leq 30(\leq 35 \%$ LOQ $)$ & 14 \\
\hline LCMS & $\leq 15(\leq 20 \%$ LOQ $)$ & 78 \\
& $\leq 20(\leq 25 \%$ LOQ $)$ & 28 \\
& $\leq 30(\leq 35 \%$ LOQ $)$ & 6 \\
\hline Hybridization ELISA & $\leq 15(\leq 20 \%$ LOQ $)$ & 13 \\
& $\leq 20(\leq 25 \%$ LOQ $)$ & 87 \\
\hline Branched DNA & $\leq 30(\leq 35 \%$ LOQ $)$ & 33 \\
\hline PCR & $\leq 30(\leq 35 \%$ LOQ $)$ & 100 \\
& Other & 50 \\
& $\leq 15(\leq 20 \%$ LOQ $)$ & 50 \\
& $\leq 20(\leq 25 \%$ LOQ $)$ & 17 \\
\hline ELISA: Enzyme-linked immunosorbent assay; HPLC: High-performance liquid chromatography; LOQ: Lower limit of quantitation; PCR: Polymerase chain reaction.
\end{tabular}

clinical and nonclinical studies has increased for $70 \%$ of these companies. The types of oligonucleotides being investigated include antisense oligonucleotides (82\%), RNA interference/siRNA (59\%), mRNA (32\%), aptamers (32\%), microRNA (23\%), CRISPR RNA (23\%) and splice switching (14\%).

All companies performing oligonucleotide assays are doing so for pharmacokinetic studies, and the majority for toxicokinetic studies as well (71\%). 29\% are assaying oligonucleotides in immunogenicity and biomarker studies. If immunogenicity is analyzed, 54\% are performing assays for modified oligonucleotides only, $15 \%$ for delivery systems and $8 \%$ for target translations. However, 39\% are assaying all of the above. The types of matrices assayed include plasma (100\%), serum (57\%), urine (38\%) and whole blood (10\%). Different types of tissues are also analyzed, including liver, kidney, lung and spleen.

An inquiry was made into the assay formats used to analyze oligonucleotides as well as the acceptance criteria applied; the results are presented in Table 1. There was discussion on whether the current regulatory guidance/guidelines are satisfactory for the analysis of oligonucleotides, with respondents equally divided for and against. Some adjust the small and large molecule guidance as needed, while others would like to see guidance for PCR platforms or tissue analysis. Only $29 \%$ of respondents have had oligonucleotide studies inspected by a regulatory agency. It was concluded during discussions that clear guidance, in the form of White Papers - or ideally, a regulatory guidance - would benefit bioanalysts engaged in oligonucleotide quantitation.

The majority of respondents $(80 \%)$ confirmed that the selected methodology met the needs of the project. When the methodology was not adequate, the reasons included sensitivity issues $(58 \%)$, regulation of tissue analysis (42\%), specificity/selectivity issues (25\%), robustness $(25 \%)$ and metabolite quantification and interference (8\%). Approximately $50 \%$ are attempting to improve their methods by switching platforms or introducing automation.

\section{'What would you do?' A session of case studies}

Three case studies were presented in order to stimulate discussion regarding typical issues facing bioanalytical CROs dealing with multiple sponsors and methods. The subjects discussed were stability assessments between laboratories, nonclinical immunogenicity and handling sponsor requests.

\section{Stability assessments between laboratories}

An assay for a prodrug was transferred from a sponsor to the CRO. The CRO had prior experience with the compound, and had data demonstrating that both the prodrug and the drug were stable in matrix at $-20^{\circ} \mathrm{C}$ for approximately 1000 days and $-70^{\circ} \mathrm{C}$ for approximately 400 days. However, because the transferred method was required by the sponsor, a new validation was conducted and stability QCs were stored at $-20^{\circ} \mathrm{C}$ to re-evaluate long-term stability. The stability of the prodrug in matrix failed at both approximately 400 days and 1100 days. Attending GCC members were asked how they would approach this situation, based on the fact that no analytical issues were found after investigation of the latest stability data or the prior stability data to justify repeating. Therefore, it was discussed whether redoing the stability for a transferred method was even required. In this case, 
the stability was not redone and the data was reported with full transparency with the caveat that stability was available from a different site. Since this data was to evaluate the secondary objective of the study, the risk was low to report in this manner.

\section{Nonclinical immunogenicity}

Bioanalysis of cynomolgus monkey samples using an anti-drug antibody (ADA) method for an antibody-drug conjugate (ADC) program was on the critical path for a biologics license application (BLA) filing. As a result, the sponsor required that all 400 samples available undergo Tier I (screening), II (confirmatory) and III (titer) analysis. In order to meet the tight deadline, three options were proposed. The first was to conduct the assay of all three tiers serially, as normal procedures dictated; however, the timing was not favorable for this option. The second option was to only conduct Tier I and II assessments simultaneously in order to determine PK anomalies. Toxicity was not observed in animals, and the drug indication was one of importance; therefore, the last option was selected, where Tier I and II data were collected simultaneously and included in the BLA filing. Then, Tier III assessments would be performed and added to an amended BLA filing. Unexpectedly, greater than $1 \%$ of the Tier I negative samples confirmed positive in Tier II. The CRO therefore decided to slow the human analysis in order to better understand the immunogenicity of the compound.

\section{Handling sponsor requests}

One of the unique challenges faced by a bioanalytical CRO is that their studies are only a piece of a greater submission package of a sponsor. Sponsors dictate deadlines and how the data is used, and this information is not always clearly available to the CRO. As a result, CROs often receive sponsor requests that seem unnecessary or to deviate from guidance without proper context, or a deficiency letter is received because a partial validation was unknowingly submitted. These requests can have a huge cost and time impact for CROs trying to minimize regulatory risk for themselves and their sponsors.

The first example was the request to limit validation exercises. Typically, full validations are required for drug filings, but the intended purpose of the method or the time available for stability studies can affect the scope of validation activities. It was concluded that all limiting factors should be included in the Validation Plan and validation reports must be very transparent as to scope. The impact of missing evaluations can be mitigated by reporting that the pending tests are time bound (e.g., stability evaluation will be performed by June 2019).

The second example of a sponsor request involved the re-assay of study samples. Several types of requests were discussed including the re-assay of study samples based on PK profile and the re-assay of samples using an alternate method.

Other sponsor requests in the panel discussion included the use of surrogate matrix for QCs and widening acceptance criteria. In all cases, it is important to work with the sponsor to understand and evaluate all available information behind their request. Examples of this include the purpose of the study (e.g., investigator study versus bioavalability/bioequivalence or comparator studies), the intended use of the data generated (e.g., experimental biomarker end point versus PK support) and the content of any related discussions held with regulatory agencies. All decisions and reporting plans should be documented a priori.

\section{Challenges of method transfer for LCMS, hybrid LBA/LCMS \& LBA}

CROs can either develop bioanalytical methods or can transfer existing methods from their sponsors or another CRO. Both avenues come with their own challenges. In order to determine the frequency of transfers versus de novo methods, and to determine the level of validation taking place for transferred methods, a survey was sent to GCC members.

Respondents use transferred methods $50 \%$ of the time or less. In general, more transfers are requested for immunoassays than for LCMS based assays. According to the CROs, the most common reasons to transfer a method are due to sponsors' internal capacity and the unique skillsets that the CROs supply. Interestingly, cost was the last reason that methods are transferred.

When a method is transferred, changes are sometimes necessary, but not always acceptable to the sponsors. For chromatography methods, changes are typically only acceptable if the method does not meet the validation criteria 'as is' (e.g., $70 \%$ of respondents report changes due to poor sensitivity) or if there are equipment/instrument platform differences (67\% of respondents). Approximately $80 \%$ of the time, changes occur to the chromatography column or conditions, or to the detector. For ligand binding methods, the reasons for changing the method during 
a transfer are largely due to poor selectivity $(65 \%)$ or a change in species $(65 \%)$. Several approaches are used, such as changing the minimum required dilution $(46 \%)$, changing the assay's range $(50 \%)$ or changing the blocking buffer (42\%).

The CRO's approach to method transfers are typically outlined in either an SOP (45\%) or a Validation Plan (39\%). Full method validation is performed by the receiving laboratory $87 \%$ of the time. When only partial validation is performed for chromatographic methods, carryover, precision, accuracy and selectivity are minimally performed at least $80 \%$ of the time, and matrix effect/factor and regression analysis are done an additional $60 \%$ of the time. For LBA, precision, accuracy and dilution linearity are performed at least $80 \%$ of the time, and selectivity $60 \%$ of the time. The remaining validation evaluations are performed less than $50 \%$ of the time.

Respondents stated that the biggest challenges in the transfer of chromatographic assays concern unclear method details or reagent availability. When questions were asked relating to what was provided by sponsors during method transfers, these positions were clarified. Analytical methods and method validation/development reports are usually included in the transfer package, as well as stable-labeled internal standards (ISs; for LCMS methods); however, relevant SOPs are almost never shared, nor are container types, which could affect nonspecific binding and adsorption. QC/stability data is only shared $64 \%$ of the time. For LBA methods, only $26 \%$ of respondents received labeled capture and detection conjugates from the sponsors. For the reagents prepared in house, $86 \%$ performed characterization experiments. In the overwhelming opinion of respondents, sharing of information was critical to the success of method transfers.

$77 \%$ of transferred methods from the sponsor to the CRO remain the property of the sponsor. Therefore, to ensure that validation meets the expectations of all, both the CRO and sponsor sign the Validation Plan $71 \%$ of the time. It was reported that transfers are successful most of the time by $60 \%$ of respondents. In fact, $74 \%$ of respondents report that the transferred methods have been successfully inspected by regulatory authorities. Those who received comments during inspections shared that when the minimum required dilution was determined by the sponsor, the original data was requested. Therefore, ensuring that method development information is available during an inspection was considered important. Additionally, an attendee was advised to always determine the cut-point during the validation of the transferred method, and not to use the sponsor supplied cut-points.

\section{Certificates of Analysis}

Reference standards play a key role in bioanalytical methods [21,22], and BMV guidance documents [19,20] require that they are appropriately characterized and documented. This documentation is provided as a COA, which must contain at least the source, lot number, expiration date, identity and purity. There are numerous providers of reference standards, and many CROs and pharma laboratories have the capability to synthesize compounds in house. Therefore, the content and quality of COAs can vary widely. The survey sent to GCC members attempted to determine how CROs are dealing with the differences; however, $66 \%$ did state that for the most part, COAs are adequate and contain all the regulatory mandated information. If not, over $60 \%$ include a deviation in a report's compliance statement. Missing information included expiration dates (69\%), impurity profile (50\%), proof of structure (38\%), storage conditions (38\%), hazards, care and handling (38\%), moisture content (31\%) and molecular weight $(12 \%)$. When receiving a sponsor-provided COA, $55 \%$ create supplementary supporting internal documentation (e.g., correction factor memo). Only $24 \%$ of respondents have ever been questioned by a regulator regarding the validity of a sponsor-provided COA. Finally, consensus was reached that a COA generated in a GMP facility can be used on a GLP study without reporting a deviation.

For small molecule bioanalysis, there are typically three types of reference standards used: certified reference standards (e.g., US pharmacopeia compendial standards) used 15\% of the time, commercially supplied reference standards obtained from a reputable source (e.g., contract manufacturing organization) used $73 \%$ of the time or other materials of documented purity custom-synthesized by an analytical laboratory or other noncommercial establishment (e.g., university) used $12 \%$ of the time.

Global BMV guidelines are not in agreement regarding the requirement of an IS COA [19,20]. Consequently, the membership is divided on this issue as well; only $51 \%$ have a requirement for a COA of an IS in their SOPs.

When it comes to determining purity, there are a number of analytical approaches. The most common methods reported by members were HPLC-UV with Karl Fisher moisture content and residue analysis (43\%), HPLC-UV with Karl Fisher moisture content only (20\%), HPLC-UV (210 nm) only (12\%), HPLC-UV (280 nm) only $(10 \%)$ or NMR (2\%). 


\begin{tabular}{|ll|}
\hline Table 2. Potential content for a macromolecule certificate of analysis. \\
\hline Requested (\%) & Elements \\
\hline$>95$ & Batch/lot number \\
& Storage conditions \\
\hline 80 & All of the above plus \\
& Retest date \\
& Expiration date \\
& Purity (280 $\mathrm{nm}$ ) \\
& Potency (activity test) \\
& Source \\
& Composition \\
\hline$>70$ & All of the above plus \\
& Known hazards, care and handling instructions \\
\hline$>60$ & All of the above plus \\
& Reconstitution stability \\
\hline$>50$ & All of the above plus \\
& Label ratios on tagged reagents \\
\hline
\end{tabular}

For macromolecules, regulators acknowledge that the test articles are heterogeneous, and their potency and immunoreactivity may vary from lot to lot [22], but that COAs should provide evidence of characterization and origin. $65 \%$ of respondents confirmed that COAs usually provide both these pieces of information. If something is missing, $70 \%$ of the time it is the characterization. Given that the understanding of the composition of the material is critical for the long-term use of a bioanalytical method (e.g., bridging in critical reagents), 95\% of respondents would like the GCC to develop a standard COA template for macromolecules to be used by both CROs and sponsors to minimize current ambiguities based on the draft WHO model that was released for comment in November 2016 [23]. Table 2 describes the desired content for such a template.

The stability of a reference standard has been discussed because both a retest date and/or an expiration date may be provided on the COA. $95 \%$ of respondents accept a COA with a retest date provided in place of an expiration date.

\section{HRMS in regulated bioanalysis}

The use of HRMS in bioanalysis has been largely limited to drug discovery [24,25]. A survey was sent to bioanalytical CROs to query the use and regulatory constraints of HRMS. Less than $40 \%$ of respondents are using HRMS in the regulated space. About 55\% of chromatography laboratories have HRMS (21\% time of flight/quad time of flight, $17 \%$ Orbitrap, $17 \%$ both). $75 \%$ of respondents expect to invest in HRMS in the next 3 years, $15 \%$ significantly. The main drivers for this investment are to begin analyzing biotherapeutics, to increase selectivity, to have the ability to collect quantitative and qualitative data simultaneously, and because sponsors are beginning to request the technology. Currently, only half of respondents have fully validated HRMS systems.

The questions regarding the utilization of HRMS indicate that the majority of bioanalysts still default to triple quadrupole instruments unless HRMS sensitivity and/or selectivity improvements are required. $45 \%$ cite cost as the main barrier to increasing implementation of HRMS throughout the industry. Regulatory uncertainty was also cited by $22 \%$ of respondents. HRMS has been primarily used for biologics. There was an approximate 50/50 mix of quantitative versus qualitative experiments, with $60 \%$ performing data mining experiments to gather qualitative information about metabolites at least occasionally.

Those laboratories that utilize HRMS use a dedicated team specially trained on the instrument. Most do not perform any different method validation tests for HRMS compared with triple quadrupole LCMS methods. The survey suggested very little interaction with regulatory authorities for HRMS submissions (only one example provided). It was concluded that any variables that may affect the assay should be addressed in the Validation Plan.

\section{Integration \& standardization of flow cytometry in bioanalysis}

Another platform on the rise in bioanalysis is flow cytometry [26]. 83\% of respondents of a survey sent to GCC membership indicated increased usage in the last 5 years. Approximately half of respondents perform flow cytometry assays, and a third of those that do not currently offer flow cytometry services plan to add them in the future. The majority of these assays are performed in a bioanalytical laboratory as opposed to in a central lab, and bioanalysts follow BMV guidance/guidelines as closely as possible. Respondents indicated that compliance is a 
key factor in deciding where to place flow cytometry studies between central and bioanalytical labs. Cell surface immunophenotyping assays are predominantly developed with this platform in order to assay mostly clinical samples.

The maximum number of colors/parameters commonly assayed is 6-8. Reagents are mostly commercially sourced. Only a third of respondents use manufacturer-recommended volumes; however, almost all perform lotto-lot bridging experiments and titration of their reagents. Typically, whole blood or PBMC samples are assayed. Healthy donors are sourced both commercially and in-house. Half of respondents use patient samples in validation. Both frozen and fixed/stabilized samples are used by $70 \%$ of respondents for staining.

Regarding the instrument, $90 \%$ of respondents have an SOP outlining maintenance requirements and processes. Moreover, many respondents require biannual preventive maintenance, although quarterly or annual preventive maintenance are also performed. Most respondents perform a daily calibration check.

Negative, positive and quality controls are typically added to each run, sourced commercially by two-thirds of respondents. When conducting validation, several parameters are evaluated. Inter and intra-assay precision runs are always performed. Also tested are sample stability (90\%), postprocessed sample stability $(80 \%)$, intrasubject variability (70\%) and sensitivity (50\%).

Software for data analysis is different from the acquisition software half of the time. The plots/gates are typically reviewed by more than two scientists. The use of automatic gating, and statistical analysis is performed only $45 \%$ of the time. GCC members attending noted that it would be beneficial to have regulatory guidance around using standard gating versus processes for adjustment based on cell population.

\section{Future perspective}

The GCC, as a global organization, will continue to provide recommendations on hot topics of global interest in small and large molecule bioanalysis, biomarkers and immunogenicity. Please contact the GCC [27] for the exact date and time of future meetings, and for all membership information.

Acknowledgments

The GCC would like to thank the following:

- N Savoie (GCC) for facilitating the 12th GCC Closed Forum.

- N Hughes (BioPharma Services), A Xu (Keystone Bioanalytical), B Stouffer (PPD), R Islam (Celerion), and M Caturla (Anapharm Bioanalytics) for chairing/speaking in the sessions on 'Recent Findings/483s'.

- P Rhyne (Immunologix Laboratories) and C Beaver (Syneos Health) for designing the survey, collecting answers, preparing survey results and chairing the session on "Critical reagent characterization and handling for both bioanalytical assays and biomarker assays".

- B Stouffer (PPD), J St Charles (Charles River) and E Tewalt (PPD) for designing the survey, collecting answers, preparing survey results and chairing the sessions on 'Oligonucleotide outsourcing: best approach for a CRO'.

- C Briscoe (PRA), E Tabler (PPD), F Spriggs (KCAS) and K Colletti (Charles River) for chairing/speaking in the sessions on 'What would you do? A session of case studies'.

- C Briscoe (PRA) and N Hughes (BioPharma Services) for designing the survey, collecting answers, preparing survey results and chairing the sessions on 'Challenges of method transfer for LCMS, hybrid LBA/LCMS and LBA'.

- R Hayes (MPI Research) for designing the survey, collecting answers, preparing survey results and chairing the session on 'Certificates of Analysis'.

- S Cape (Covance) and P Bennett (PPD) for designing the survey, collecting answers, preparing survey results on 'HRMS in regulated bioanalysis'.

- P Bennett (PPD) and V Kakkanaiah (PPD) for chairing the session on 'Integration and standardization of flow cytometry in bioanalysis'.

- R Islam (Celerion) for designing the survey, collecting answers, preparing survey results and chairing the session on 'Classification of commercial kits to support regulated bioanalysis'.

- M Khan (previously at Alliance Pharma, currently at TEVA) for helping designing the survey on 'Classification of commercial kits to support regulated bioanalysis'.

- All the GCC member companies who filled in the numerous surveys, participated and contributed to the discussions at the 12th GCC Closed Forum for Bioanalysis. 
- N Savoie (GCC) for taking the minutes of the 12th GCC Closed Forum and drafting the first draft of this document.

- W Garofolo (GCC) for organizing the logistics of the meeting and coordinating the review of this document.

Financial \& competing interests disclosure

The authors have no relevant affiliations or financial involvement with any organization or entity with a financial interest in or financial conflict with the subject matter or materials discussed in the manuscript. This includes employment, consultancies, honoraria, stock ownership or options, expert testimony, grants or patents received or pending, or royalties.

No writing assistance was utilized in the production of this manuscript.

\section{References}

1. Premkumar N, Lowes S, Jersey J et al. Formation of a Global Contract Research Organization Council for bioanalysis. Bioanalysis 2(11), 1797-1800 (2010).

2. Breda N, Garofolo F, Cruz Caturla M et al. The 3rd Global CRO Council for Bioanalysis at the International Reid Bioanalytical Forum. Bioanalysis 3(24), 2721-2727 (2011).

3. Lowes S, Jersey J, Shoup R et al. 4th Global CRO Council for Bioanalysis: coadministered drugs stability, EMA/US FDA guidelines, 483s and carryover. Bioanalysis 4(7), 763-768 (2012).

4. Nicholson R, Lowes S, Caturla MC et al. 6th GCC focus on LBA: critical reagents, positive controls and reference standards; specificity for endogenous compounds; biomarkers; biosimilars. Bioanalysis 4(19), 2335-2342 (2012).

5. Rocci M, Lowes S, Shoup R et al. 7th GCC: use of incurred samples; fit-for-purpose validation; solution stability; electronic lab notebooks; hyperlipidemic matrix testing. Bioanalysis 6(20), 2713-2720 (2014).

6. Bower J, Fast D, Garofolo F et al. 8th GCC: consolidated feedback to US FDA on the 2013 draft FDA guidance on bioanalytical method validation. Bioanalysis 6(22), 2957-2963 (2014).

7. Hayes R, LeLacheur R, Dumont I et al. 9th GCC closed forum: CAPA in regulated bioanalysis; method robustness, biosimilars, preclinical method validation, endogenous biomarkers, whole blood stability, regulatory audit experiences and electronic laboratory notebooks. Bioanalysis 8(6), 487-495 (2016).

8. Cape S, Islam R, Nehls C et al. The 10th GCC Closed Forum: rejected data, GCP in bioanalysis, extract stability, BAV, processed batch acceptance, matrix stability, critical reagents, ELN and data integrity and counteracting fraud. Bioanalysis 9(7), 505-516 (2017).

9. Lowes S, Jersey J, Shoup R et al. Recommendations on: internal standard criteria, stability, incurred sample reanalysis and recent 483 s by the Global CRO Council for Bioanalysis. Bioanalysis 3(12), 1323-1332 (2011).

10. Boterman $M$, Doig $M$, Breda $M$ et al. Recommendations on the interpretation of the new EMA guideline on bioanalytical method validation by Global CRO Council for Bioanalysis (GCC). Bioanalysis 4(6), 651-660 (2012).

11. Sangster T, Maltas J, Struwe P et al. Recommendations on ISR in multi-analyte assays, QA/bioanalytical consultants and GCP by Global CRO Council for Bioanalysis (GCC). Bioanalysis 4(14), 1723-1730 (2012).

12. Lowes $\mathrm{S}$, Boterman M, Doig M et al. Recommendations on bioanalytical method stability implications of co-administered and co-formulated drugs by Global CRO Council for Bioanalysis (GCC). Bioanalysis 4(17), 2117-2126 (2012).

13. Houghton R, Gouty D, Allinson J et al. Recommendations on biomarker bioanalytical method validation by GCC. Bioanalysis 4(20), 2439-2446 (2012).

14. Lowes S, LeLacheur R, Shoup R et al. Recommendations on incurred sample stability (ISS) by GCC. Bioanalysis 6(18), 2385-2390 (2014).

15. Islam R, Briscoe C, Bower J et al. 11th GCC Closed Forum: cumulative stability; matrix stability; immunogenicity assays; laboratory manuals; biosimilars; chiral methods; hybrid LBA/LCMS assays; fit-for-purpose validation; China Food and Drug Administration bioanalytical method validation. Bioanalysis 10(7), 433-444 (2018).

16. Islam R, Kar $\mathrm{S}$, Ritzén $\mathrm{H}$ et al. Recommendations for classification of commercial immunoassay kits for biomarkers in drug development from the Global CRO Council Bioanalysis 11(7), 645- 653 (2019).

17. Ministerio da Saude Resolução da Diretoria Colegiada - RDC No 27, de 17 de Maio de 2012 (2012). http: //portal.anvisa.gov.br/documents/33880/2568070/rdc0027_17_05_2012.pdf/c6edeb56-200d-4482-8a19-99fa11c33fd3

18. Gupta S, Richards S, Amaravadi L et al. 2017 White Paper on recent issues in bioanalysis: a global perspective on immunogenicity guidelines \& biomarker assay performance (Part 3 - LBA: immunogenicity, biomarkers and PK assays). Bioanalysis 9(24), 1967-1996 (2017).

19. European Medicines Agency. Committee for medicinal products for human use (CHMP). Guideline on bioanalytical method validation. London, UK (2011). EMEA/CHMP/EWP/192217/2009.

20. US Department of Health and Human Services, US FDA, Center for Drug Evaluation and Research, Center for Veterinary Medicine. Draft Guidance for Industry, Bioanalytical Method Validation, Rockville, MD, USA (2013). 
21. DeSilva B, Garofolo F, Rocci M et al. 2012 White Paper on recent issues in bioanalysis and alignment of multiple guidelines. Bioanalysis 4(18), 2213-2226 (2012).

22. Stevenson L, Amaravadi L, Myler H et al. 2014 White Paper on recent issues in bioanalysis: a full immersion in bioanalysis (Part 3 - LBA and immunogenicity). Bioanalysis 6(24), 3355-3368 (2014).

23. WHO. Draft model certificate of analysis. Geneva, Switzerland (2016).

24. Welink J, Fluhler E, Hughes N et al. 2015 White Paper on recent issues in bioanalysis: focus on new technologies and biomarkers (Part 1 - small molecules by LCMS). Bioanalysis 7(22), 2913-2925 (2015).

25. Yang E, Welink J, Cape S et al. 2016 White Paper on recent issues in bioanalysis: focus on biomarker assay validation (BAV) (Part 1 small molecules, peptides and small molecule biomarkers by LCMS). Bioanalysis 8(22), 2363-2378 (2016).

26. Richards S, Amaravadi L, Pillutla $R$ et al. 2016 White Paper on recent issues in bioanalysis: focus on biomarker assay validation (BAV): (Part 3 - LBA, biomarkers and immunogenicity). Bioanalysis 8(23), 2475-2496 (2016).

27. Global CRO Council for Bioanalysis. Global CRO Council homepage (2019). www.global-cro-council.org

Authors

Chad Briscoe 1 , Nicola Hughes², Roger Hayes ${ }^{3}$, Rafiq Islam4 ${ }^{4}$ Patrick Bennett ${ }^{5}$, Bruce Stouffer ${ }^{5}$, Stephanie Cape ${ }^{6}$, Paul Rhyne ${ }^{7}$, Chris Beaver ${ }^{8}$, Jessica St Charles ${ }^{3}$, Vellalore Kakkanaiah ${ }^{5}$, Allan Xu ${ }^{9}$, Maria Cruz Caturla ${ }^{10}$, Franklin Spriggs ${ }^{11}$, Rabab Tayyem ${ }^{12}$, Colin Barry ${ }^{13}$, Anahita Keyhani ${ }^{14}$, Jennifer Zimmer $^{15}$, Philippe Couerbe ${ }^{16}$, Mark Warren ${ }^{16}$, Ardeshir Khadang ${ }^{17}$, James Bourdage ${ }^{18}$, Kathie Lindley ${ }^{19}$, Dave Williams ${ }^{19}$, Curtis Sheldon ${ }^{4}$, Christina Satterwhite ${ }^{20}$, Jenifer Vija ${ }^{21}$, Mathilde $\mathrm{Yu}^{22}$, Iohann Boulay ${ }^{23}$, John Stamatopoulos ${ }^{23}$, Jenny Lin ${ }^{24}$, Sian Estdale ${ }^{25}$, Eric Thomas ${ }^{26}$, Andrew Dinan ${ }^{27}$, Robert MacNeill ${ }^{28}$, Yi Qun Xiao 28 , Luca Matassa ${ }^{29}$, Wei Garofolo*30, Natasha Savoie ${ }^{30}$, George Hristopoulos $^{31}$, Arron Xu ${ }^{31}$, Lawrence Goodwin ${ }^{11}$, Kayode Awaiye ${ }^{32}$, Hanna Ritzén ${ }^{33}$, Mohammed Bouhajib ${ }^{34}$, Chantal Di Marco ${ }^{34}$, Simona Rizea Savu ${ }^{35}$, Corey Nehls ${ }^{5}$, Edward Tabler ${ }^{5}$, Amanda Hays ${ }^{1}$, Shane Karnik ${ }^{36}$, Michael Brown ${ }^{37}$, Steve Lowes ${ }^{38}$, Ira DuBey ${ }^{39}$, Nadia Kulagina ${ }^{39}$, John Lindsay ${ }^{40}$, Clark Williard ${ }^{8}$, Hao Wang ${ }^{41}$, Michele Malone ${ }^{42}$, Edward Wells ${ }^{42}$, Xinping Fang ${ }^{43}$ \& Marc Moussallie ${ }^{43}$

\section{Affiliations}

1 PRA Health Sciences, Lenexa, KS, USA

${ }^{2}$ BioPharma Services, 4000 Weston Road, North York, Toronto, M9L

3A2 Ontario, Canada

${ }^{3}$ MPI Research, Mattawan, MI 49071, USA

${ }^{4}$ Celerion, Lincoln, Nebraska, NE 68502, USA

${ }^{5}$ PPD Laboratories, Richmond, VA 23230, USA

${ }^{6}$ Covance, Madison, WI 53704, USA

${ }^{7}$ Immunologix Laboratories, Tampa, FL 33634, USA

${ }^{8}$ Syneos Health, Princeton, NJ 08540, USA

${ }^{9}$ Keystone Bioanalytical, North wales, PA 19454, USA

${ }^{10}$ Anapharm Bioanalytics, Barcelona 08038, Spain

${ }^{11}$ KCAS Bioanalytical \& Biomarker Services, Shawnee Mission, KS 66216, USA

${ }^{12}$ ACDIMA Center for Bioequivalence \& Pharmaceutical Studies, Amman, Jordan

${ }^{13}$ Alliance Pharma, Malvern, PA 19355, USA

${ }^{14}$ Altasciences, Laval, Quebec H7V 4B3, Canada

${ }^{15}$ Alturas Analytics, Moscow, ID 83843, USA

${ }^{16}$ Atlanbio, Route de Saint-André des Eaux, Saint-Nazaire 44600, France

${ }^{17}$ Axis Clinicals, Dilworth, MN 56529, USA

${ }^{18}$ BASi, West Lafayette, IN 47906, USA

${ }^{19}$ BioAgilytix, Durham, NC 27713, USA

${ }^{20}$ Charles River Laboratories, Reno, NV 89511, USA
${ }^{21}$ Charles River Laboratories, Skokie, IL 60077, USA

${ }^{22}$ CIRION Biopharma Research, Laval, Quebec H7L 5E1, Canada

${ }^{23}$ CiToxLAB North America, Laval, Quebec H7V 4B3, Canada

${ }^{24}$ CMIC, Hoffman Estates, IL 60192, USA

${ }^{25}$ Covance, Harrogate, HG3 1 PY, UK

${ }^{26}$ Covance, Indianapolis, IN 46214, USA

${ }^{27}$ CPR Pharma Services, Adelaide, SA 5031, Australia

${ }^{28}$ Envigo, Somerset, NJ 08873, USA

${ }^{29}$ Frontage Lab, Exton, PA 19341, USA

${ }^{30}$ Grand Challenges Canada, Toronto, Ontario M5G 1M1, Canada

${ }^{31}$ Intertek, San Diego, CA 92121, USA

${ }^{32}$ Lambda Therapeutic Research, Toronto, Ontario M1L 4S4, Canada

${ }^{33}$ Mercodia AB, Uppsala 754 50, Sweden

${ }^{34}$ Pharma Medica Research Inc., Mississauga, Ontario L5R 0B7, Canada

${ }^{35}$ Pharma Serv International, Bucharest 050853, Romania

${ }^{36}$ Pyxant Labs, Colorado Springs, CO 80907, USA

${ }^{37} \mathrm{Q}^{2}$ Solutions, Marietta, GA 30067, USA

${ }^{38} \mathrm{Q}^{2}$ Solutions, Ithaca, NY 14850, USA

${ }^{39}$ Smithers Avanza, Gaithersburg, MD 20878, USA

${ }^{40}$ SNBL USA, Everett, WA 98203, USA; currently part of Altasciences

${ }^{41}$ TETRAQ, Herston, QLD 4029, Australia

${ }^{42}$ Worldwide Clinical Trials, Austin, TX 78754, USA

${ }^{43}$ WuXi Apptec, Plainsboro, NJ 08536, USA 